\title{
A novel, scalable, and modular bioreactor design for dynamic simulation of the digestive tract
}

\author{
Salam Habib ${ }^{1}$, Anikka Swaby ${ }^{1}$, Stan Kubow ${ }^{1}$, and Luis Agellon ${ }^{1}$ \\ ${ }^{1}$ McGill University - MacDonald Campus
}

February 24, 2021

\begin{abstract}
In vitro gut model systems permit the growth of gut microbes outside their natural habitat and are essential to the study of gut microbiota. Systems available today are limited by lack of scalability and flexibility in mode of operation. Here we describe the development of a versatile bioreactor module capable of sensing and controlling of environmental parameters such as $\mathrm{pH}$ control of culture medium, rate of influx and efflux of the culture medium, and aerobic/anaerobic atmosphere. Modules can be linked in series to construct a model of a digestive tract to allow the growth of microbiota in vitro. We tested the growth of a model bacterial community in a simulated mammalian gut model. The model attained and maintained a stable bacterial community that metabolized bile acids. The findings illustrate the utility of the model to grow to culture a mixed bacterial community and recapitulate biological activities such as bile acid metabolism in vitro.
\end{abstract}

\section{A novel, scalable, and modular bioreactor design for dynamic simulation of the digestive tract}

Salam Habib | Anikka M. Swaby | Stan Kubow | Luis B. Agellon

School of Human Nutrition, McGill University, Ste. Anne de Bellevue, QC, H9X 3V9 Canada

Running title: Modular bioreactor for culture of gut bacteria

\section{Correspondence}

Luis B. Agellon, School of Human Nutrition, McGill University, 21111 Lakeshore Road, Ste. Anne de Bellevue, QC H9X 3V9 Canada.

E-mail: luis.agellon@mcgill.ca

\section{Funding information}

Natural Sciences and Engineering Council of Canada, Discovery Grants RGPIN-2015-04390 and RGPIN2020-04901.

\section{Abstract}

In vitro gut model systems permit the growth of gut microbes outside their natural habitat and are essential to the study of gut microbiota. Systems available today are limited by lack of scalability and flexibility in mode of operation. Here we describe the development of a versatile bioreactor module capable of sensing and controlling of environmental parameters such as $\mathrm{pH}$ control of culture medium, rate of influx and efflux of the culture medium, and aerobic/anaerobic atmosphere. Modules can be linked in series to construct a model of a digestive tract to allow the growth of microbiota in vitro. We tested the growth of a model bacterial community in a simulated mammalian gut model. The model attained and maintained a stable bacterial community that metabolized bile acids. The findings illustrate the utility of the model to grow to 
culture a mixed bacterial community and recapitulate biological activities such as bile acid metabolism in vitro.

\section{KEYWORDS}

In vitro gut model, artificial gut, microbiota, continuous culture

\section{1 | INTRODUCTION}

The gut microbiota is a diverse and complex community of bacteria, archaea, fungi and viruses. The characteristics of its species composition and function have garnered great interest of researchers in recent years. The microbial community is an integral part of the gastrointestinal tract as it provides the host with an extended set of metabolic, structural and protective functions (O'Hara \& Shanahan, 2006). In recent times, dysbiosis of the gut microbiota has been associated with a variety of diseases, such as inflammatory bowel diseases, diabetes, obesity, cancers, and even some behavioral disorders (Musso et al., 2011; Manichanh et al., 2012; Zhu et al., 2013; Vuong et al., 2017). It is now accepted that the gut microbiota has a strong influence on host health.

Due to the complex nature of the host-microbial interaction, it is difficult to infer specific mechanistic aspects of this interaction using conventional in vivo models and thus highlights the need for facile in vitro systems to help understand the complex interactions between the host and its gut microbiota (de Vos \& de Vos, 2017). The currently available in vitro gut models vary in their complexity (Molly et al, 1993; Minekus et al., 1999; Makivuokko \& Nurminen, 2006; Payne et al., 2012; Tanner et al., 2014; Moon et al., 2016; Card et al., 2017; von Martels et al., 2017; Dupont et al., 2019). Batch culture models are simple closed fermentation systems, that usually operate for less than two days. In continuous culture models, which might be single or multi-stage in design, fresh culture medium, supplemental growth factors or any other additives, are added continuously, while wastes and toxic byproducts are prevented from accumulating excessively by continuous dilution of the spent medium with fresh culture medium (Moon et al., 2016). The addition of fresh medium can be scheduled at specified time intervals as in the semi-continuous model EnteroMix (Makivuokko \& Nurminen, 2006), or continuously supplied as in the Simulator of the Human Intestinal Microbial Ecosystem (SHIME) model (Molly et al, 1993). Some models, such as the TNO Gastro-Intestinal Model TIM1 and TIM2 systems (Minekus et al., 1999), permit simulation of intestinal tract processes like digestion and absorption. Other models permit incorporation of intestinal tissues by employing co-culturing techniques (von Martels et al., 2017). Different gut models have been developed for in vitro study of complex microbial communities associated with various physiological and pathological states, or from the digestive tracts of humans and animals (Tanner et al., 2014; Card et al., 2017).

Since most of the in vitro gut models in existence were designed to simulate a specific digestive tract or condition, their designs inherently make it difficult to study different conditions or applications using the one device. Here we describe the design and implementation of a modular bioreactor. The novel bioreactor design serves as a new platform that features unparalleled functional flexibility for the culturing of monoand mixed bacterial cultures in vitro. In particular, the bioreactors can operate either as single static batch cultures or connected in series to dynamically simulate the environments of the various segments of the gastrointestinal tract.

\section{2 | MATERIALS AND METHODS}

\section{1 | System design}

The basic component of the gut model is a standardized bioreactor module (Figure 1). The growth chamber (clear or brown media bottles with GL45 caps; Corning Incorporated) is placed in a jacketed reaction beaker (Chemglass) that serves as the temperature control vessel and is set on top of a stir plate. The temperature control vessel is attached to a recirculating heater/chiller with Prestone ${ }^{\circledR}$ antifreeze/engine coolant as the heat transfer fluid. The growth chamber is equipped with a stir bar and the caps are drilled to accommodate dedicated ports for a pH sensor (Atlas Scientific, Long Island City, NY), inlet and outlet ports for transfer of culture medium, inlet ports for acid and base, inlet port for nitrogen gas, outlet port for venting excess gas, 
and a valved outlet port for culture sampling. Brown bottles offer protection of light sensitive metabolites from photodamage. The space between the growth chamber and the temperature control vessel is filled with Prestone ${ }^{\circledR}$ antifreeze/coolant to ensure efficient heat transfer. The nitrogen gas inlet can be adapted to permit either an aerobic (port open to the room atmosphere) or anaerobic (constant flow of nitrogen gas) culture environment as required. Mini-peristaltic pumps (Kamoer KPP-DH-B03W), which convey acid $(\mathrm{HCl})$ or base $(\mathrm{NaOH})$ to the growth chamber, and larger peristaltic pumps (New Era NE-9000), which drive the transfer of culture medium from one growth chamber to the next, are all controlled by a Raspberry Pi computer version B-R2 (Raspberry Pi Foundation) running the system control software (Figure S1) written in Python programming language (Python Software Foundation). All the computers controlling each of the modules are connected via an ethernet router. The $\mathrm{pH}$ probe is connected to the Raspberry Pi computer through an $\mathrm{EZO}^{\mathrm{TM}} \mathrm{pH}$ circuit stamp (Atlas Scientific, Long Island City, NY). A time server running on a dedicated Raspberry Pi computer fitted with a real-time clock module synchronizes the clocks across all network devices. The number of bioreactor modules operated at a time is flexible (Figure S2). Batch culture mode (growth medium In/Out ports sealed), continuous mode (single bioreactor with growth medium with In/Out ports operational) and series mode can be selected at the start of the experiment and the system parameters are supplied to each control computer through a configuration file.

\subsection{Starter culture and culture conditions}

The model gut microbiota in this study was prepared from dehydrated bacterial cultures of Lactobacillus acidophilus, Lactobacillus delbrueckii subspecies bulgaricus, Lactobacillus casei, Lactobacillus rhamnosus, Bifidobacterium bifidum, Bifidobacterium breve, Bifidobacterium longum and Streptococcus salivarius subspeciesthermophilus. Some experiments used monocultures ofEscherichia coli BL21 or Bifidobacterium longum subspeciesinfantis 35624 (Bifantis, Align). To establish working bacterial cultures, dehydrated bacteria were first reconstituted in sterile peptone water (Oxoid). A $1 \mathrm{~mL}$ aliquot of the reconstituted bacteria was then added to $9 \mathrm{~mL}$ of de Man Rogosa and Sharpe (MRS) medium (Oxoid) supplemented with $0.05 \% \mathrm{w} / \mathrm{v}$ L-cysteine (Amresco). The starter culture was grown at $37 \operatorname{deg} C$ anaerobically until the optical density at $600 \mathrm{~nm}\left(\mathrm{OD}_{600}\right)$ reached [?] 1.5 before being used in experiments.

\section{3 | DNA extraction and quantification}

Bacterial cells were sedimented from $1 \mathrm{~mL}$ cultures by centrifugation. The pellet was resuspended in buffer containing $0.2 \mathrm{M} \mathrm{NaCl}, 0.1 \mathrm{M}$ ethylenediaminetetraacetic acid (EDTA) and $15 \mathrm{mg} / \mathrm{mL}$ lysozyme, and then incubated at $37 \mathrm{deg} \mathrm{C}$ for $60 \mathrm{~min}$. SST solution (20\% sarkosyl, $0.2 \mathrm{M} \mathrm{NaCl}, 0.5 \mathrm{M}$ Tris- $\mathrm{HCl} \mathrm{pH} 8.0,1 \mathrm{mg} / \mathrm{mL}$ proteinase $\mathrm{K}$ and $4 \mathrm{mM}$ dithiothreitol) was then added and the samples were further incubated at $55 \mathrm{degC}$ for $60 \mathrm{~min}$. Nucleic acids were extracted with an equal volume of phenol:chloroform (1:1) solvent. The aqueous phase was recovered, and nucleic acids were precipitated with 2 volumes of cold absolute ethanol in the presence of $0.3 \mathrm{M}$ sodium acetate, washed with cold $70 \%$ ethanol, dried and then finally dissolved in buffer containing $5 \mathrm{mM}$ Tris-HCl $(\mathrm{pH} 8.0)$ and $0.1 \mathrm{mM}$ EDTA. The concentration of DNA was determined using the Qubit ${ }^{(\mathrm{r})}$ dsDNA BR Assay Kit (Invitrogen). The integrity of the isolated DNA was assessed by agarose gel electrophoresis.

\section{4 | Biochemical analyses}

Quantitative real-time PCR (qPCR) reactions were carried out using a Bio-Rad CFX96 real-time system operated by CFX Manager software (version 3.1). The standard reaction mixture consisted of $80 \mathrm{mM}$ Tris$\mathrm{HCl} \mathrm{pH} \mathrm{8.3,} 100 \mathrm{mM} \mathrm{KCl}, 6 \mathrm{mM} \mathrm{MgCl} 2,300 \mathrm{mM}$ trehalose, $200 \mathrm{mM}$ betaine- $\mathrm{HCl}, 0.2 \mathrm{mg} / \mathrm{mL}$ bovine serum albumin, $0.2 \%$ (v/v) Tween-20, 1 X SYBR Green I, $2 \mathrm{mM}$ dNTP mix, $0.4 \mathrm{U} / \mu \mathrm{L}$ Taq polymerase, $5 \mu \mathrm{M}$ each of forward and reverse primers and $1 \mu \mathrm{g} / \mathrm{mL}$ DNA template in a total volume reaction volume of 10 $\mu \mathrm{L}$. The sequences of the primers used in this study is shown in Table S1. The standard PCR reaction included an initial denaturation step of $95{ }^{\circ} \mathrm{C}$ for 5 min and 40 cycles of $95{ }^{\circ} \mathrm{C}$ for $10 \mathrm{~s}$ and annealing and extension at $65{ }^{\circ} \mathrm{C}$ for $30 \mathrm{~s}$, followed by melt curve analysis from $65{ }^{\circ} \mathrm{C}$ to $95{ }^{\circ} \mathrm{C}$ in $0.5{ }^{\circ} \mathrm{C}$ increments. Results were expressed as proportions of the copy number determined for each target within the sample, and each sample was represented as a percentage of the culture with the highest bacterial density which was assigned 
an arbitrary value of $100 \%$.

Bile acids were separated by high performance liquid chromatography (HPLC) as described previously (Torchia et al., 2001). Bile acid species were identified based on retention times of certified bile acid standards (Steraloids) and quantified against known amounts of the bile acid standards using area under the curve method.

\section{5 | Statistical analyses}

Differences between means were evaluated by Student's $t$-tests using GraphPad Prism software version 6.01 for Windows (GraphPad Software). Differences were considered significant when $P<0.05$.

\section{3 | RESULTS AND DISCUSSION}

\subsection{Aerobic and anaerobic environment}

To evaluate the ability of the bioreactors to maintain aerobic and anaerobic conditions, two bioreactor modules were inoculated with a monoculture of the anaerobe Bifidobacterium longum subspeciesinfantis (B. infantis) and operated in the batch culture mode. This bacterium was able to grow and thrive as indicated by the increase in the $\mathrm{OD}_{600}$ of the culture after $24 \mathrm{~h}$ (Figure 2A). Analysis of the composition of the culture by qPCR confirmed that the increase in bacterial density over the $24 \mathrm{~h}$ of culture was due to the growth of B. infantis (Figure 2B). In a separate experiment, a set of bioreactor modules were inoculated with a mixed bacterial culture containing different species of bifidobacteria along with other bacterial species from two of the major phyla (Firmicutes and Actinobacteria) of the human gut microbiota (Kostic et al., 2013) to enable us to assess the growth dynamics of bifidobacteria within a mixed bacterial community. In this experiment, the MRS culture medium (Lee \& Lee, 2008; Süle et al., 2014) was used and the bioreactor modules were operated in the continuous culture mode. As shown in Figure $2 \mathrm{C}$, the mixed culture maintained under anaerobic condition showed higher growth rates compared to that maintained under aerobic condition. Moreover, the bacterial growth under anaerobic culture condition was attributable to the growth of Bifidobacterium spp . and Streptococcus spp. at the expense of the Lactobacillus spp. (Figure 2D). These results demonstrated the ability of the bioreactor modules to maintain anaerobic culture conditions and support the growth of anaerobic bacteria such as bifidobacteria.

\section{$3.2 \mid \mathrm{pH}$ control}

To assess the efficacy of $\mathrm{pH}$ control, six bioreactor modules were operated independently in batch culture mode. All bioreactor modules were inoculated with an active E. coli BL21 culture. One group had no pH control (Figure 3A) and the other group was set to maintain $\mathrm{pH} 7$ (Figure 3B). The pH of the cultures was monitored for $24 \mathrm{~h}$ and samples of the cultures were collected for assessment of optical density at regular intervals over the course of the experiment. The cultures of $E$. coli maintained at pH 7 showed enhanced growth during the exponential growth phase compared to the cultures without $\mathrm{pH}$ control. The volume of acid and base added to the growth medium to maintain pH 7 are shown in Figure 3C. These results demonstrated the ability of the bioreactor modules to maintain a steady $\mathrm{pH}$ of the culture at a predetermined value.

\subsection{Genetic stability of a model mixed bacterial community in culture}

To assess the genetic stability of the bacterial community in long-term culture, the bioreactors were connected in series to create a dynamic model of the human digestive tract. In this experiment, six bioreactor modules were connected in series, each set to maintain a specific $\mathrm{pH}$ and held under anaerobic conditions to simulate a segment of the human gastrointestinal tract (Figure 4A). The $\mathrm{pH}$ values used in the stomach, small intestine, and large intestine compartments were based on the $\mathrm{pH}$ values reported for these regions of the human digestive tract (Khutoryanskiy, 2015). At the start of the experiment, Module 1 (representing the stomach) was inoculated with the model microbiota as described earlier (Figure 4B) and the system was operated continuously for $120 \mathrm{~h}$. As shown in Figure 4C-4H, bacterial density in Module 1 decreased (Figure $4 \mathrm{C}$ ), while the bacterial density in Modules 2 and 3 (representing the proximal and distal small intestine, respectively; Figures 4D and 4E) as well as Modules 4-6 (representing the ascending colon, transverse colon 
and descending colon of the large intestine, respectively; Figures $4 \mathrm{~F}-4 \mathrm{H})$ increased over the duration of the experiment. Modules 4-6 exhibited the greatest bacterial density. At the end of the experiment, the genetic composition of the bacterial community in Module 6, which simulates the most distal large intestine compartment (Figure 4H), closely resembled that of the initial inoculum (Figure 4B) at the genus level. The specific bacterial species and their abundance in each of the bioreactor modules in the series were also determined. The composition of the bacterial community was distinct at different $\mathrm{pH}$ represented by the different segments of the digestive tract (Figure 4A). B. bifidum was not detectable after $24 \mathrm{~h}$ of culture, suggesting that specific nutritional or structural substrate requirements of this particular species that may have not been met by the culture conditions. In contrast, L. casei, L. rhamnosus, B. longum, and $S$. thermophilus remained easily detectable throughout the entire duration of the experiment (Figure S3).

\subsection{Assessment of biological activity of the model mixed bacterial community}

To test if the model gut microbial community grown in the in vitro gut model exhibited selected biological activities associated with gut microbiota in vivo, the ability of the culture to metabolize bile acids was assayed. In the first experiment, six bioreactor modules operating in the batch culture mode with $\mathrm{pH}$ control were inoculated with the model microbiota. Taurocholic acid (TCA), a common conjugated bile acid, was added to the culture medium at 0,10 , or $100 \mathrm{mM}$ concentrations. The growth of the model microbiota treated with TCA was substantially less compared to the untreated model microbiota. Nevertheless, bacterial growth was observed even at the highest TCA concentration used in the experiment (Figure 5A). At $10 \mathrm{mM}$ TCA treatment, the proportion ofStreptobacteria spp. in the community was reduced while that ofLactobacillus spp. was increased (Figure 5B), which is likely due to its ability to metabolize TCA (Gilliland \& Speck, 1977). HPLC analysis of the bile acid species in the culture medium showed the appearance of cholic acid, a product of TCA deconjugation by bacteria, after $24 \mathrm{~h}$ of culture (Figure $5 \mathrm{C}$ ). In the second experiment, the model microbiota was treated with chenodeoxycholic acid (CDCA), an unconjugated bile acid. CDCA was more potent than TCA in inhibiting the growth of the model microbiota (Figure 5D). As shown in Figure 5E, ursodeoxycholic acid, the $7 \beta$-hydroxyl group epimer of the $7 \alpha$-hydroxyl group-containing CDCA produced by bacterial metabolism, was present in the culture after $24 \mathrm{~h}$. These results demonstrated that the model microbiota grown in the in vitro digestive tract model could exhibit biological activities normally associated with healthy gut microbiota in vivo.

\section{4 | CONCLUSIONS}

We described here the design and validation of a modular bioreactor module that can be operated under various configurations. Each module can sense and control the $\mathrm{pH}$ of its culture medium and can be set to operate in static or dynamic culturing mode to serve specific applications. The modular bioreactor design offers flexibility in terms of culture size, aerobic/anaerobic atmosphere, and static/dynamic culture. Specifically, the bioreactor design enables the creation of simple or complex models of digestive tracts of desired culture sizes. The resulting systems can be used to study the growth and evolution of cultured microbes, such as gut microbes, independent of host immune surveillance as well as to determine the response of the microbial community to controlled availability of specific metabolites that are normally secreted by the host into the digestive tract.

\section{ACKNOWLEDGMENTS}

This work was supported by the Discovery Grants from the Natural Sciences and Engineering Council of Canada (RGPIN-2015-04390 to LBA; RGPIN-2020-04901 to SK).

\section{CONFLICT OF INTERESTS}

None declared.

\section{AUTHOR CONTRIBUTIONS}

SH and AMS assembled hardware components, wrote software, performed experiments, analyzed the data and wrote the manuscript. SK and LBA developed the bioreactor design, designed experiments, analyzed 
the data, wrote and edited the manuscript.

\section{ORCID}

Luis B. Agellon (ID) https://orcid.org/0000-0002-2209-8702

\section{REFERENCES}

Card, R. M., Cawthraw, S. A., Nunez-Garcia, J., Ellis, R. J., Kay, G., Pallen, M. J., Woodward, M. J., and Anjum, M. F. (2017). An in vitro chicken gut model demonstrates transfer of a multidrug resistance plasmid from Salmonella to commensal Escherichia coli, mBio, 8, e00777-17. https://doi.org/10.1128/mBio.00777-17

de Vos, W. M. and de Vos, E. A. (2012). Role of the intestinal microbiome in health and disease: from correlation to causation, Nutrition Reveiws, 70, S45-56. https://doi.org/10.1111/j.1753-4887.2012.00505.x

Dupont, D., Alric, M., Blanquet-Diot, S., Bornhorst, G. Cueva, C., Deglaire, A., Denis, S. Ferrua, M., Havenaar, R., Lelieveld, J., Mackie, A. R., Marzorati, M., Menard, O., Minekus, M., Miralles, B., Recio, I., Van den Abbeele, P. (2019). Can dynamic in vitro digestion systems mimic the physiological reality? Critical Reveiws in Food Science and Nutrition, 59, 1546-1562. https://doi.org/10.1080/10408398.2017.1421900

Gilliland, S. E. and Speck, M. L (1977). Deconjugation of bile acids by intestinal lactobacilli, Applied and Environmental Microbiology, 33, 15-18. https://doi.org/10.1128/AEM.33.1.15-18.1977

Kostic, A. D., Howitt, M. R., and Garrett, W. S. (2013). Exploring host-microbiota interactions in animal models and humans, Genes and Development, 27, 701-718. https://doi.org/10.1101/gad.212522.112

Khutoryanskiy, V. V. (2015). Supramolecular materials: Longer and safer gastric residence, Nature Materials, 14, 963-964. https://doi.org/10.1038/nmat4432

Lee, H. M. and Lee, Y. (2008). A differential medium for lactic acid-producing bacteria in a mixed culture, Letters in Applied Microbiology, 46, 676-681. https://doi.org/10.1111/j.1472-765X.2008.02371.x

Makivuokko, H. and Nurminen, P. (2006). In vitro methods to model the gastrointestinal tract. In Ouwehand, A. and Vaughan, E. (Eds.), Gastrointestinal Microbiology. Boca Raton, FL: Taylor \& Francis Group.

Manichanh, C., Borruel, N., Casellas, F., and Guarner, F. (2012). The gut microbiota in IBD, Nature Reviews Gastroenterology \& Hepatology, 9, 599-608. https://doi.org/10.1038/nrgastro.2012.152

Minekus, M., Smeets-Peeters, M., Havenaar, R., Bernalier, A., Fonty, G., Marol-Bonnin, S., Alric, M., Marteau, P., and Huis in't Veld, J. H. J. (1999). A computer-controlled system to simulate conditions of the large intestine with peristaltic mixing, water absorption and absorption of fermentation products, Applied Microbiology and Biotechnology, 53, 108-114. https://doi.org/10.1007/s002530051622

Molly, K., Vande Woestyne, M., and Verstraete, W. (1993). Development of a 5-step multi-chamber reactor as a simulation of the human intestinal microbial ecosystem, Applied Microbiology and Biotechnology, 39, 254-258. https://link.springer.com/article/10.1007\%2FBF00228615

Moon, J. S., Li, L., Bang, J., and Han, N. S. (2016). Application of in vitro gut fermentation models to food components: A review, Food Science and Biotechnology, 25, 1-7. https://doi.org/10.1007/s10068-016-0091-x

Musso, G., Gambino, R., and Cassader, M. (2011). Interactions between gut microbiota and host metabolism predisposing to obesity and diabetes, Annual Review of Medicine, 62, 361-380. https://doi.org/10.1146/annurev-med-012510-175505

O'Hara, A. and Shanahan, F. (2006). The gut flora as a forgotten organ, EMBO Reports, 7, 688-693. https://doi.org/10.1038/sj.embor.7400731

Payne, A. N., Zihler, A., Chassard, C., and Lacroix, C. (2012). Advances and perspectives in in vitro human gut fermentation modeling, Trends in Biotechnology, 30, 17-25. https://doi.org/10.1016/j.tibtech.2011.06.011 
Süle, J., Kõrösi, T., Hucker, A., and Varga, L. (2014). Evaluation of culture media for selective enumeration of bifidobacterial and lactic acid bacteria, Brazilian Journal of Microbiology, 45, 1023-1030. https://doi.org/10.1590/s1517-83822014000300035

Torchia, E. C., Labonte, E. D., and Agellon, L. B. (2001). Separation and quantitation of bile acids using an isocratic solvent system for high performance liquid chromatography coupled to an evaporative light scattering detector, Analytical Biochemistry, 298, 293-298. https://doi.org/10.1006/abio.2001.5379

Tanner, S. A., Zihler Berner, A., Rigozzi, E., Grattepanche, F., Chassard, C., and Lacroix, C. (2014). In vitro continuous fermentation model (PolyFermS) of the swine proximal colon for simultaneous testing on the same gut microbiota, PLoS One, 9, e94123. https://doi.org/10.1371/journal.pone.0094123

von Martels, J. Z. H., Sadaghian Sadabad, M., Bourgonje, A. R., Blokzijl, T., Dijkstra, G., Faber, K. N., and Harmsen, H. J. M. (2017). The role of gut microbiota in health and disease: In vitro modeling of host-microbe interactions at the aerobe-anaerobe interphase of the human gut, Anaerobe, 44, 3-12. https://doi.org/10.1016/j.anaerobe.2017.01.001

Vuong, H. E., Yano, J. M., Fung, T. C., and Hsiao, E. Y. (2017). The microbiome and host behavior, Annual Review of Neuroscience, 40, 21-49. https://doi.org/10.1146/annurev-neuro-072116-031347

Zhu, Q. C., Gao, R. Y., Wu, W., and Qin, H. L. (2013). The role of gut microbiota in the pathogenesis of colorectal cancer, Tumor Biology, 34, 1285-1300. https://doi.org/10.1007/s13277-013-0684-4

\section{SUPPORTING INFORMATION}

This manuscript has additional supporting information.

\section{FIGURE LEGENDS}

FIGURE 1 Schematic representation of a bioreactor module.

FIGURE 2 Aerobic and anaerobic growth of a gut bacteria. (A) Growth of Bifidobacterium longum subspecies infantis (B. infantis) monocultures under anaerobic conditions at $\mathrm{pH}$ 5.8. Data shown is the average of two trials with $n=4$ replicates. (B) Abundance of bacteria detected by the bacterial 16S rDNA universal primers and 16S rDNA primers specific for Bifidobacterium longum in equivalent masses of input template DNA extracted from the monocultures in (A). Data shown is the average of two trials with $\mathrm{n}=4$ replicates. (C) Growth of a model gut microbiota (mixed bacterial cultures) over time in aerobic and anaerobic conditions maintained at $\mathrm{pH}$ 5.8. Data shown is the average of two trials with $\mathrm{n}=2$ replicates. (D) Proportions of bacterial genera present in the starter culture $(0 \mathrm{~h})$ and after $24 \mathrm{~h}$ of growth in aerobic and anaerobic cultures $(\mathrm{C}) . *{ }^{*}<0.05$.

FIGURE 3 Growth of a bacterial monocultures with and without $\mathrm{pH}$ control. Growth of an E. coli monocultures over time (A) without $\mathrm{pH}$ control and (B) at $\mathrm{pH}$ 7.0. (C) Total volume of acid and base added to the $\mathrm{pH}$-controlled culture (B) over time. Data shown is the average of 2 trials with $n=3$ replicates.

FIGURE 4 Stability of the genotype of a model gut microbiota in a dynamic simulation of the human digestive tract. (A) The $\mathrm{pH}$ setpoint of the culture medium representing segments of the simulated digestive tract. The rate of influx and efflux of culture medium into and out of each bioreactor was set to $0.1 \mathrm{~mL} / \mathrm{min}$. (B) Proportions of bacterial genera of the starter culture. The growth of the model gut microbiota over 5 days and the proportions of bacterial genera assayed at 24, 72, $120 \mathrm{~h}$ in modules representing (C) Stomach (Module 1), (D) Proximal small intestine (Module 2), (E) Distal small intestine (Module 3), (F) Ascending colon (Module 4), (G) Transverse colon (Module 5) and (H) Descending colon (Module 6). The areas of the circles depict the density of the bacterial cultures at the time of sampling with the density of the inoculum (B) set to $100 \%$. Data shown is the average of 2 trials with $n=2$ replicates.

FIGURE 5 Functionality of a model gut microbiota in response to bile acid treatment. (A) Growth of a model gut microbiota in the anaerobic conditions at $\mathrm{pH} 5.8$ in the absence or presence of taurocholic acid (TCA). Data shown is the average of two trials with $n=2$ replicates. (B) Proportions of bacterial genera 
present at inoculation $(0 \mathrm{~h})$ and after $24 \mathrm{~h}$ of culture (A). (C) Representative chromatogram showing the detection of TCA and cholic acid (CA) in the growth medium of inoculated cultures at $0 \mathrm{~h}$ and $24 \mathrm{~h}$, and uninoculated culture (no bacteria) at $24 \mathrm{~h}$, in the presence of $10 \mathrm{mM}$ TCA. (D) Growth of a model gut microbiota in the presence of chenodeoxycholic acid (CDCA). Data shown is the mean of 2 trials with $\mathrm{n}=4$ replicates. (E) Detection of CDCA and ursodeoxycholic acid (UDCA) in the growth medium at inoculation $(0 \mathrm{~h})$ and after $24 \mathrm{~h}$ of culture. ND, not detected.

FIGURE S1 Algorithm of the bioreactor control software.

FIGURE S2 Example configurations of simulated gut models.

FIGURE S3 Species composition of the bacterial community present in specific bioreactor modules of the dynamic simulation of the human digestive tract as described in the legend of FIGURE 4.

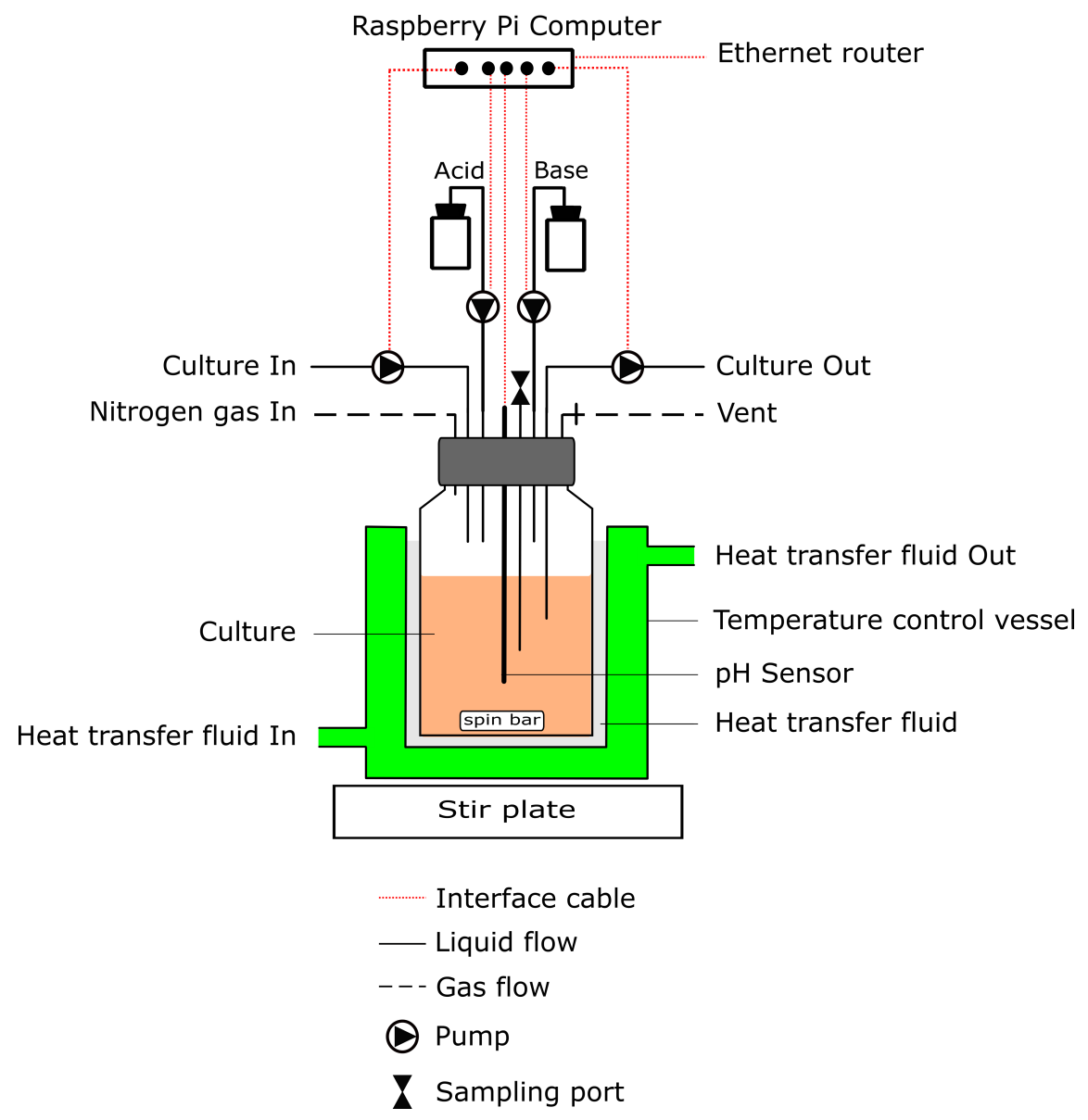


A

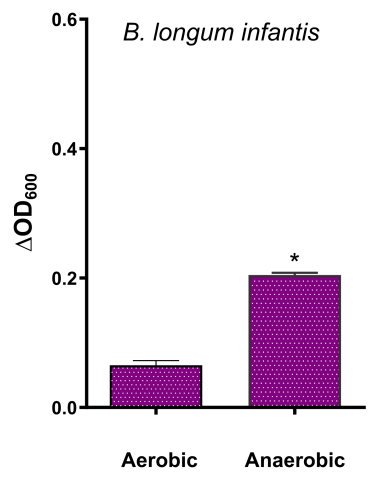

C

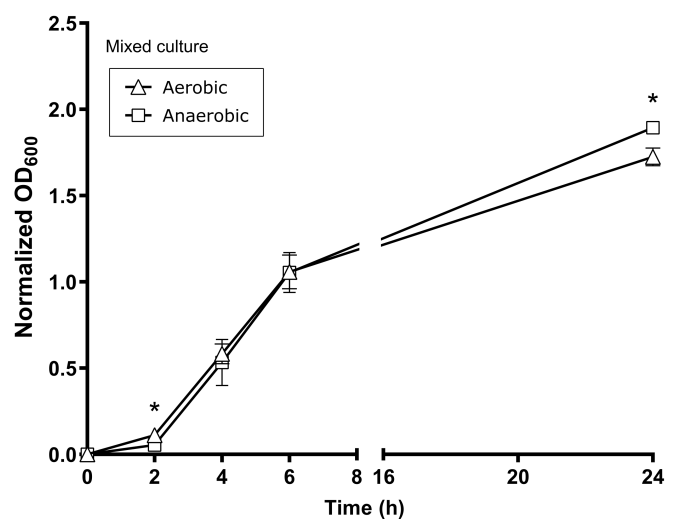

B

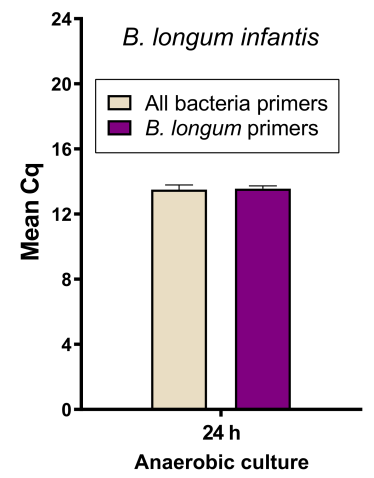

D

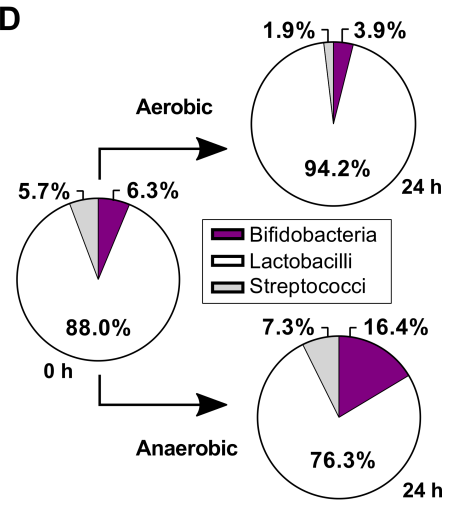


A

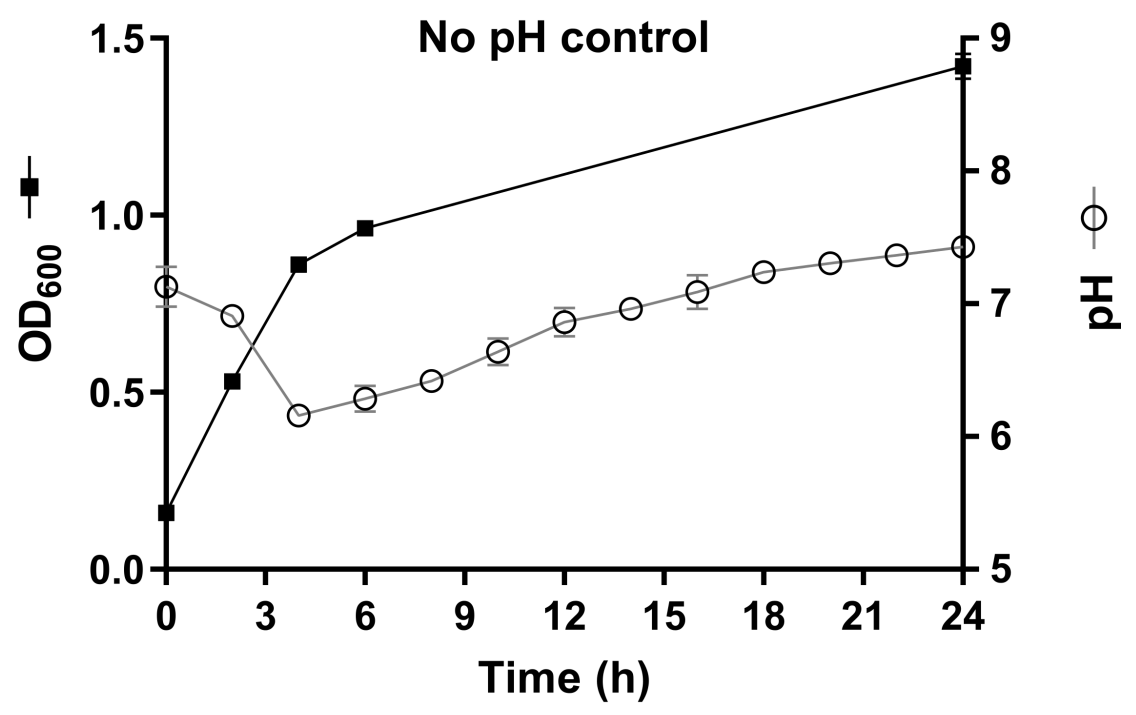

B

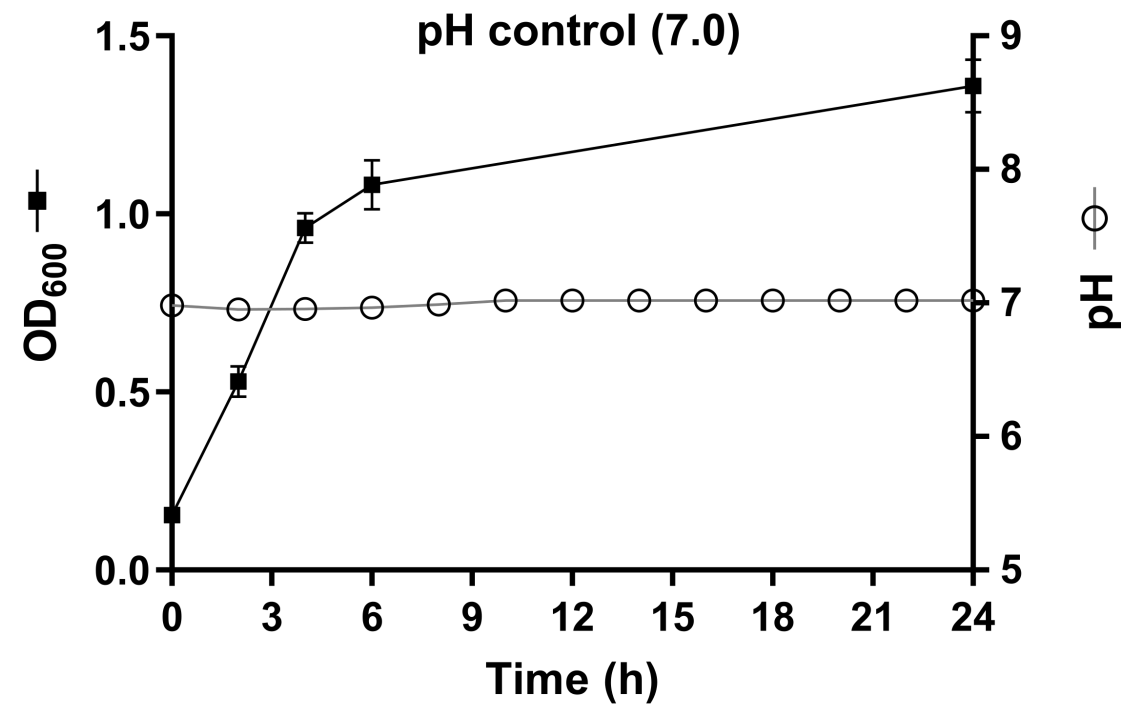

C

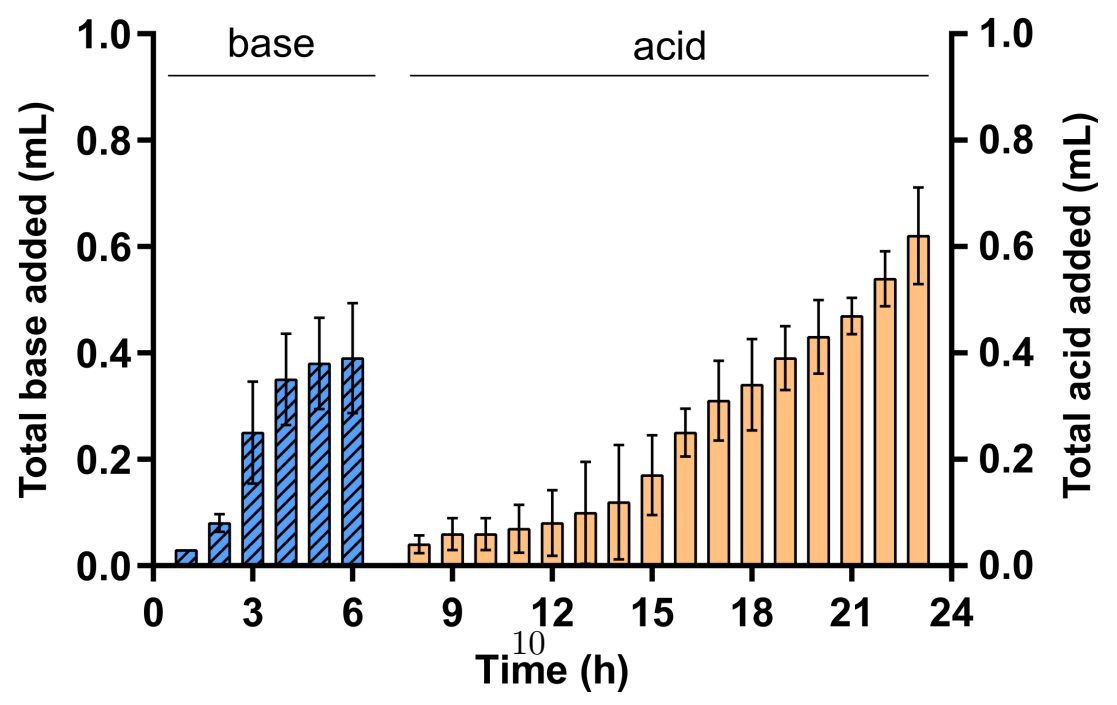



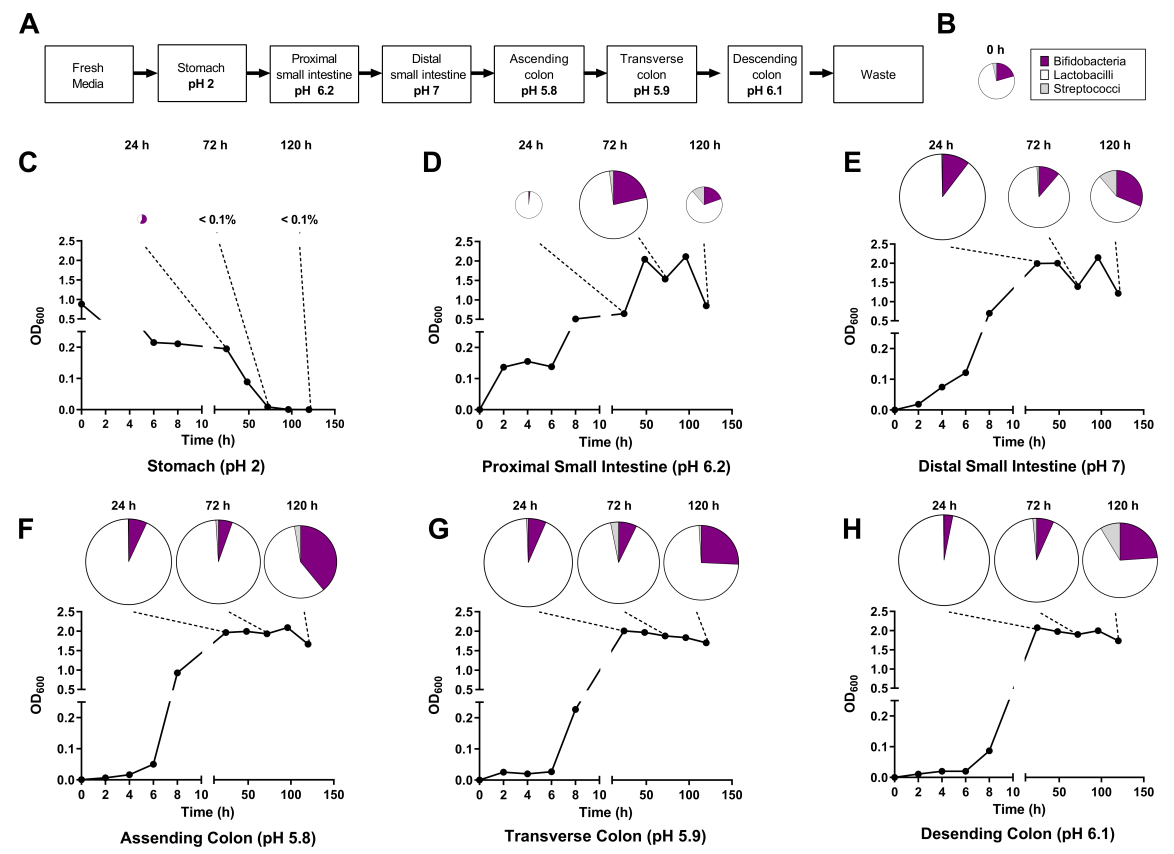
A

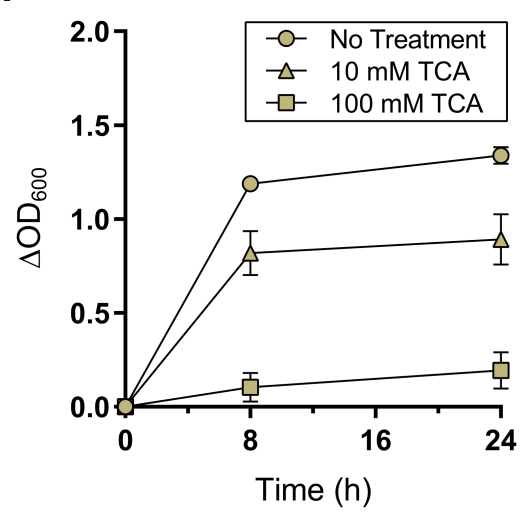

C

TCA

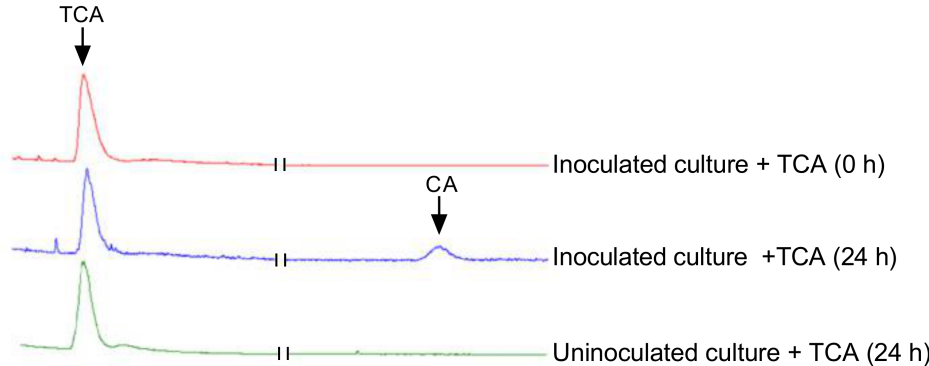

D

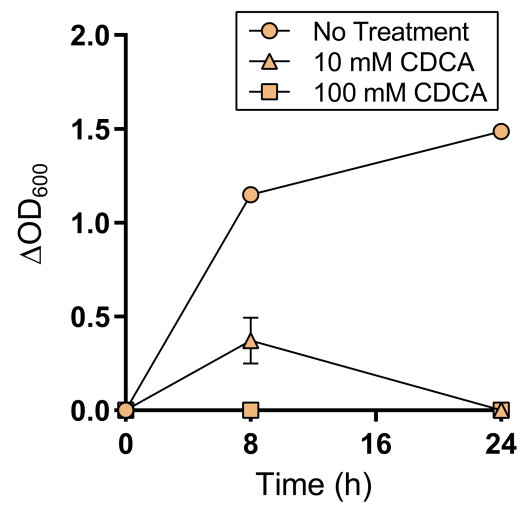

\section{E}

B

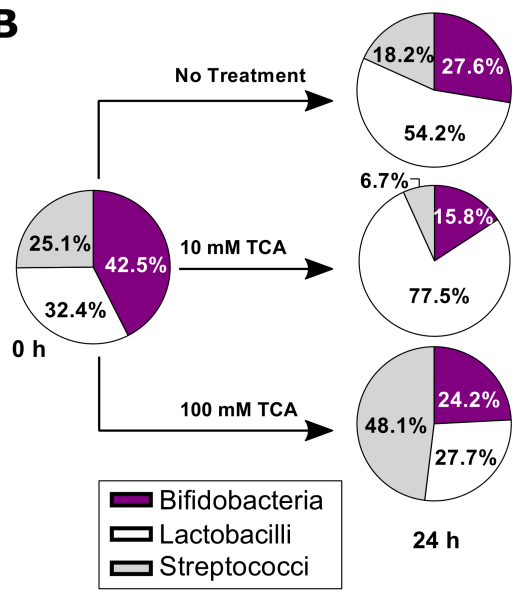

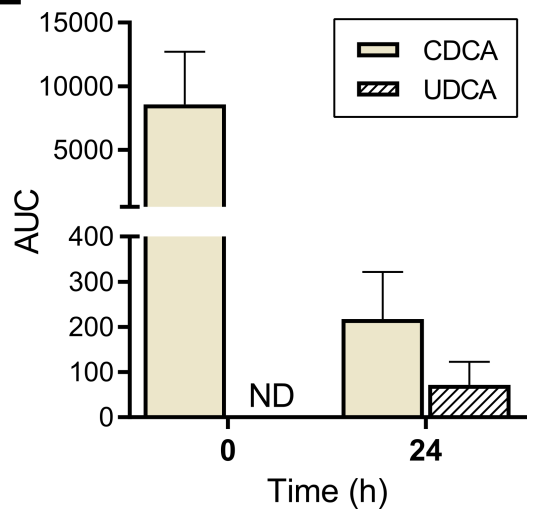

